\title{
Endovascular rescue of vertebro-basilar thrombosis in cervical spine injury
}

\author{
U Ratnasabapathy ${ }^{1} \cdot$ M Purcell $^{2} \cdot J$ J Bhattacharya ${ }^{3,4}$
}

Received: 1 October 2018 / Revised: 13 October 2018 / Accepted: 16 October 2018

(c) International Spinal Cord Society 2018

\begin{abstract}
Introduction Vertebro-basilar thrombosis is often lethal. We report a post-traumatic case in which logistical issues were overcome to secure prompt endovascular intervention resulting in a favourable outcome.

Case presentation We report this case to highlight (i) the need, across the United Kingdom, for rapid access to 24/7 neurointerventional services and (ii) the fact that vertebral artery injury during C-spine trauma, while not uncommon, can rarely have catastrophic complications.

Discussion Vertebral artery injuries during C-spine trauma can rarely present as a neurovascular emergency requiring neurointerventional rescue. It is our opinion that patients with neurovascular emergencies merit 24/7 neurointerventional services, akin to those provided for acute coronary syndromes.
\end{abstract}

\section{Introduction}

The setting of this case was in the Queen Elizabeth National Spinal Injuries Unit (QENSIU), Glasgow, UK. With the patient's consent, we extracted information from their records and imaging in order to report the case.

\section{Case presentation}

A 29-year-old male tried to hang upside down by his legs from a pull-up bar that gave way while he was exercising. He fell about 2 feet onto his neck and shoulders, with his head in forward flexion. Without loss of consciousness and within 90 min of injury, he was taken by ambulance to the local emergency department. On arrival, he was alert with

\footnotetext{
U Ratnasabapathy

Urmila.ratnasabapathy@nhs.net

1 Department of Neuroanaesthesia, Queen Elizabeth University Hospital, Glasgow, Scotland

2 National Spinal Injuries Unit, Queen Elizabeth University Hospital, Glasgow, Scotland

3 King Faisal Specialist Hospital and Research Center and Alfaisal University, Riyadh, Saudi Arabia

4 Department of Neuroradiology, Queen Elizabeth University Hospital, Glasgow, Scotland
}

normal respiratory and cardiovascular parameters but was unable to retain information normally or comply with bed rest. He had severe neck pain but neither headache nor visual symptoms. He could move all limbs but had tingling in the $\mathrm{C} 7$ dermatome of the fingers bilaterally. He retained the ability to void urine under voluntary control.

Initial computed tomography (CT) (Figs. 1 and 2) showed cervical fractures at $\mathrm{C} 4$ and $\mathrm{C} 5$ with disruption of the anterior longitudinal ligament, a burst fracture of $\mathrm{C} 6$, a large para-vertebral haematoma, and step misalignment of 3 $\mathrm{mm}$ at the $\mathrm{C} 6 / 7$ level with slight narrowing of the canal. No other injuries were apparent on $\mathrm{CT}$ of head, abdomen, and pelvis or the skeletal survey.

Eighteen hours after injury, the patient was transferred to the QENSIU using our standard spinal precautions. On admission, his finger paraesthesia had receded but he was complaining of neck and low back pain. Neurological examination confirmed reduced $\mathrm{C} 7$ reflexes and reduced power at 4 of 5 in the elbow extensors. Pin-prick sensation was altered in the $\mathrm{T} 1$ dermatomes. He was classified according to the International Standards for Neurological Classification of Spinal Cord Injury (SCI) as C6 incomplete tetraplegia AIS (ASIA Impairment Scale) D. After magnetic resonance imaging (MRI) of the neck (Fig. 3), a neurosurgical consult judged there was no indication for emergency spinal fixation. The patient was immobilised on an Atlas spinal bed using our standard precautions. 


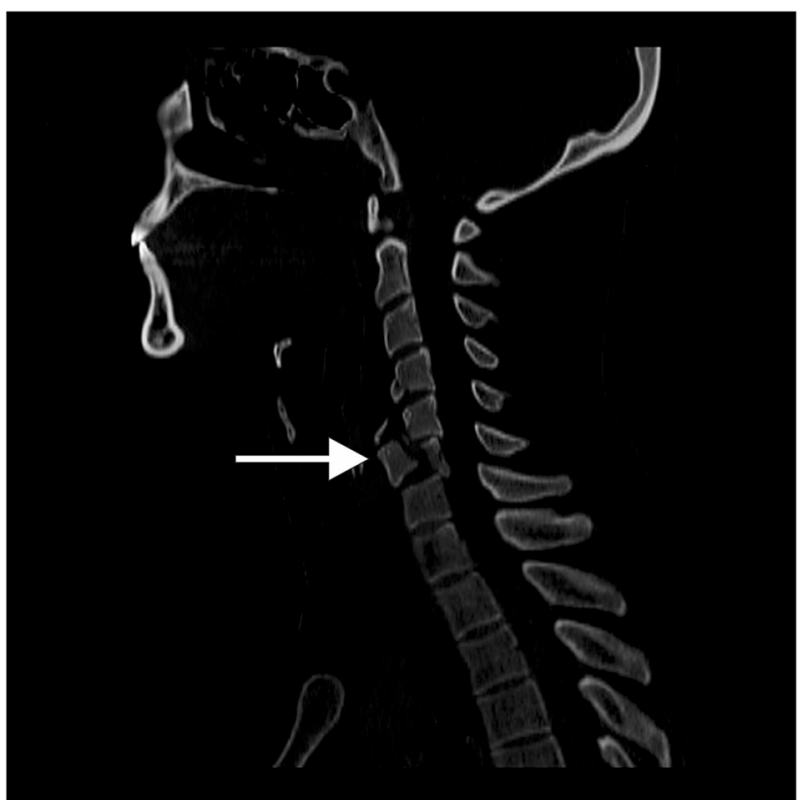

Fig. 1 Sagittal reconstruction CT cervical spine. Fracture dislocation is demonstrated at C6 (arrow)

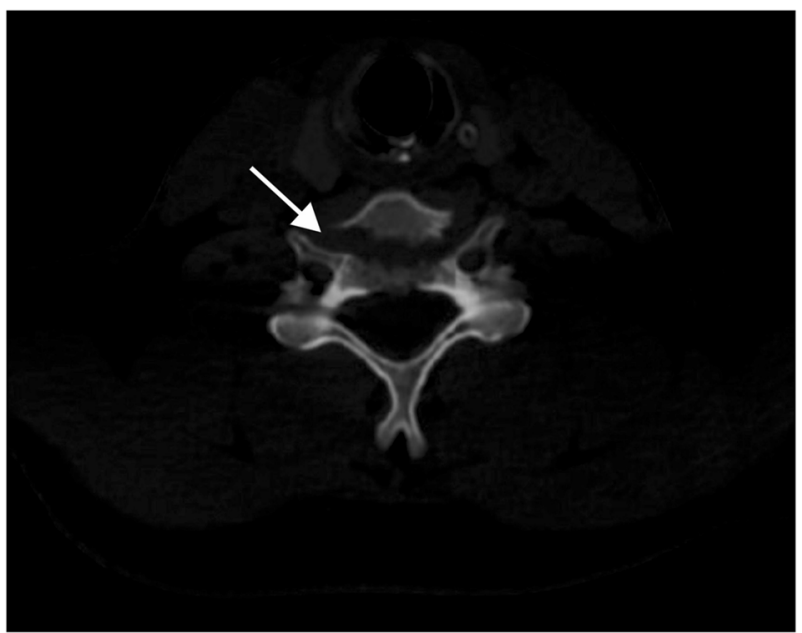

Fig. 2 Axial view C6 vertebra. Fracture line runs through vertebral body (arrow)

Twenty-four hours after admission to QENSIU, the patient deteriorated rapidly. He began breathing noisily and, although his eyes remained open, became unresponsive to pain. An oropharyngeal airway was inserted. His respiratory and cardiovascular status remained stable. His pupils were enlarged, one not reacting to light. After $2 \mathrm{~min}$, he began responding to pain. Within a further $2 \mathrm{~min}$, he was responding to questions, but was noted now to be dysarthric with a new, dense, left-sided hemiparesis. Urgent CT head and CT Angiogram confirmed a posterior circulation stroke due to bilateral vertebral artery occlusion with mid-vertebral artery dissection and basilar artery occlusion (Fig. 4).

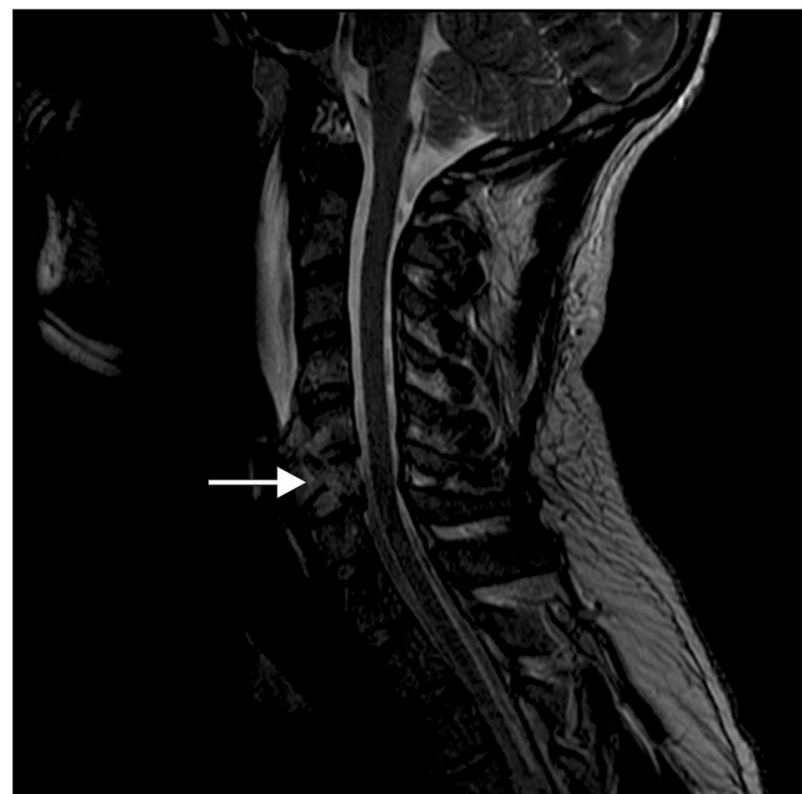

Fig. 3 MRI neck, sagittal T2-weighted imaging. There is no evidence of spinal cord compression

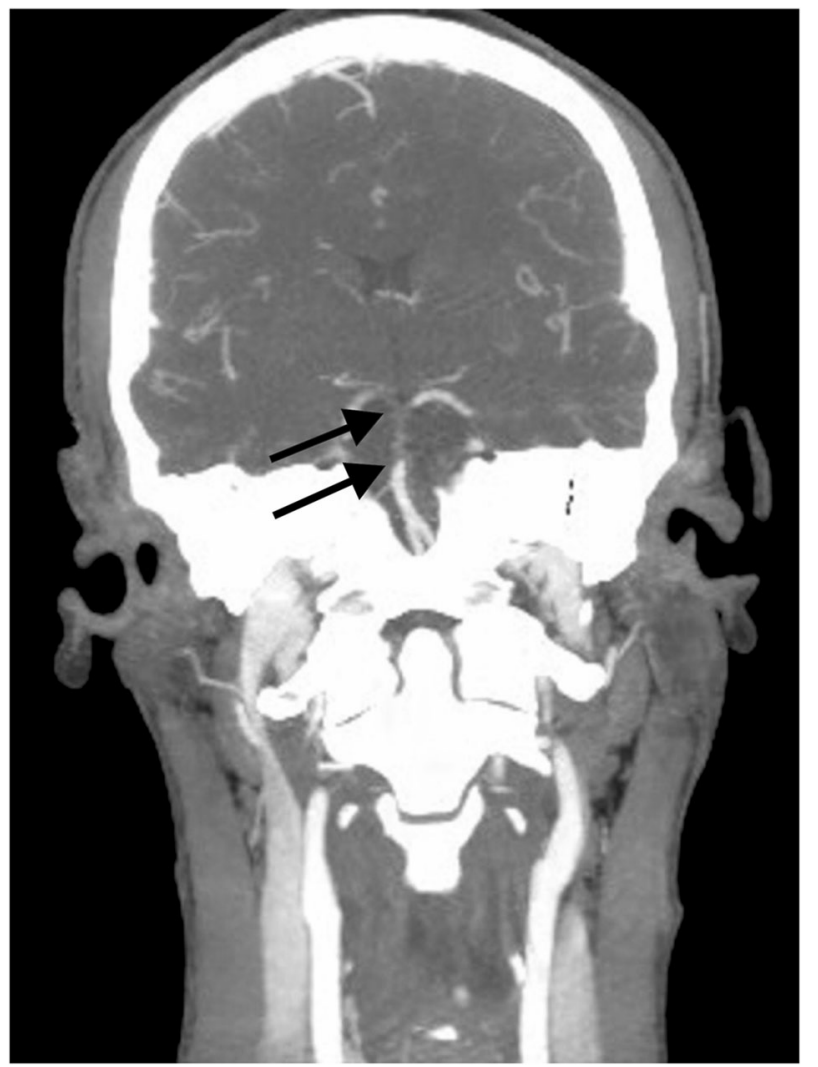

Fig. 4 CT Angiography demonstrating filling defect within basilar artery, indicating basilar occlusion (arrows) 


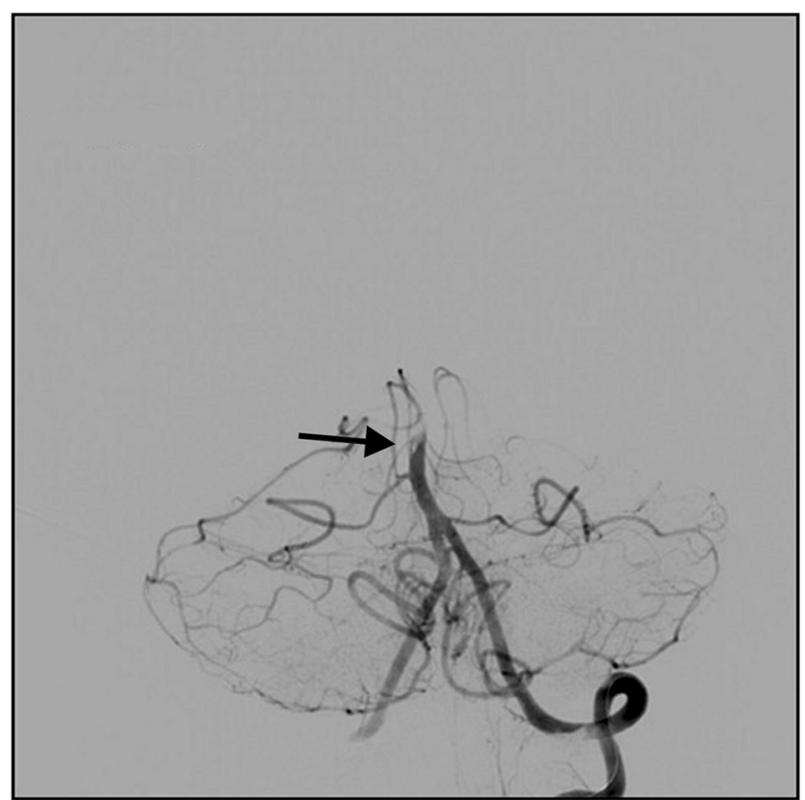

Fig. 5 Digital subtraction angiography. Confirms embolic occlusion of the mid-basilar artery (arrow)

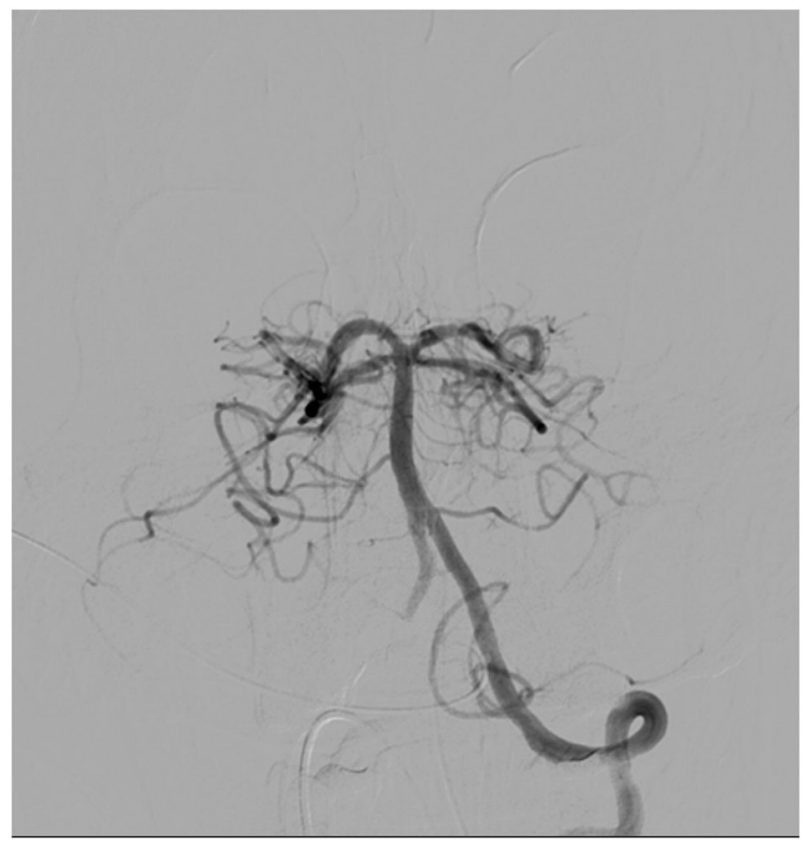

Fig. 6 Digital subtraction angiography. The embolus was removed by a single pass of a stentriever thrombectomy device. Angiographic run confirms restored flow in the basilar artery

Intravenous thrombolysis (tissue Plasminogen Activator (tPA) bolus and infusion) was started while in the CT scanner and continued on the ward. Despite tPA infusion, his neurological status worsened over $90 \mathrm{~min}$. Only an 'incomprehensible sounds' verbal response could be elicited on the Glasgow Coma Score.
In Scotland, there is no funded out-of-hours service for neurointervention post stroke. However, on the weekend of this episode, our Neurosciences Institute was on call for aneurysm coiling for Scotland. Given the abrupt deterioration, the patient was reviewed by a stroke physician and a neurointerventional radiologist was contacted to attempt emergency clot retrieval. By this time, the patient was only eye opening spontaneously, making incomprehensible sounds and localising to pain.

His head and neck stabilised in-line; the patient's trachea was intubated with the aid of muscle relaxation and indirect laryngoscopy with a McGrath laryngoscope. Angiography (Figs. 5 and 6) showed normal filling of the anterior circulation and the distal posterior communicating artery territories. There was no filling of the basilar territory from the anterior circulation. The right vertebral artery was occluded approximately $2 \mathrm{~cm}$ above its origin. The left vertebral artery had reopened since the intravenous tPA. There was a dissection flap at mid-left vertebral artery but no obstruction to flow. A $2 \mathrm{~cm}$ thrombus was successfully retrieved from the basilar artery, leaving only small residual clot in the right vertebral artery. A heparin infusion was started.

Post thrombectomy, the patient was transferred to NeuroICU, then QENSIU. He was moving all limbs. The need for anticoagulation meant external fixation with Bremmer Halo was preferred to surgical management for his spinal fracture.

MRI head was undertaken 5 days post endovascular treatment to gauge the impact of this treated vertebro-basilar thrombosis. It showed small infarcts in the right medial thalamus, both sides of the pons and both cerebellar hemispheres. Two and three weeks post injury, the patient scored 92/100 on Addenbrookes's Cognitive Examination and has had further improvement since.

The patient was eventually discharged home with family support. On discharge from QENSIU, he was independently mobile and self-caring. His elbow extension weakness was still evident but had improved causing no functional impairment.

Warfarin anticoagulation was prescribed for 6 months. Halo fixation was removed in clinic, 3 months after being applied. At this time his neurology was normal and there was no neck pain. A Miami-J collar was fitted. A lateral Cspine X-ray 2 weeks later showed no change and no kyphosis at the fracture site. Seven months later, the patient had returned to work full time as a mechanic.

\section{Discussion}

Several randomised trials have demonstrated the positive effect of endovascular thrombectomy in the treatment of large vessel ischaemic stroke. At least four positive trials 
published in 2015 [1-4] have led to guideline changes in the United States. Of note, basilar artery occlusion was excluded from these trials [5].

We have reported the endovascular rescue of a young man, from a lethal complication of cervical trauma. Although single cases do not guarantee best policy, we highlight two issues from this case that should not be neglected.

First, we would propose that all UK stroke patients deserve 24/7 access to neurointerventional rescue on the two grounds of benefit and equity. In terms of benefit, neurointervention looks to have averted a catastrophic outcome and facilitated a return to work without the major care costs of long-term disability. In regard of equity, stroke patients deserve similar access to endovascular rescue that has transformed the lives of those having stents for acute coronary syndromes or coiling of cerebral aneurysms.

Second, we would argue that although vertebral artery injury is a well reported (often inconsequential) feature of cervical spine injury, clinicians need to retain a keen awareness of the complication reported here, because rapid endovascular rescue may avert death or major disability.
Acknowledgements We thank Dr Natasha Fullarton, Consultant Neuroradiologist, Queen Elizabeth Hospital, Glasgow, for reviewing the imaging.

\section{Compliance with ethical standards}

Conflict of interest The authors declare that they have no conflict of interest.

\section{References}

1. Berkhemer OA, Fransen PS, Beumer D, van den Berg LA, Lingsma $\mathrm{HF}$, Yoo AJ. et al. A randomized trial of intraarterial treatment for acute ischemic stroke. N Engl J Med. 2015;372:11-20.

2. Campbell BC, Mitchell PJ, Kleinig TJ, Dewey HM, Churilov L, Yassi N. et al. Endovascular therapy for ischemic stroke with perfusion-imaging selection. N Engl J Med. 2015;372:1009-18.

3. Goyal M, Demchuk AM, Menon BK, Eesa M, Rempel JL, Thornton J. et al. Randomized assessment of rapid endovascular treatment of ischemic stroke. N Engl J Med. 2015;372:1019-30.

4. Jovin TG, Chamorro A, Cobo E, di Miquel MA, Molina CA, Rovira A. et al. Thrombectomy within 8 hours after symptom onset in ischemic stroke. N Engl J Med. 2015;372:2296-306.

5. Campbell BCV, Donnan GA, Mitchell PJ, Davis SM. Endovascular thrombectomy for stroke: current best practice and future goals. Stroke Vasc Neurol 2016;1:16-22 https://doi.org/10.1136/svn2015-000004 